\title{
Analyse de la structure de peuplements ligneux dans un « fourré tigré » au nord Yatenga (Burkina Faso). État actuel et conséquences évolutives
}

\author{
P Couteron ${ }^{1}$, A Mahamane ${ }^{1}$, P Ouedraogo ${ }^{2}$ \\ ${ }^{1}$ Département forêts des régions chaudes, Engref, 34033 Montpellier; \\ ${ }^{2}$ Laboratoire d'écologie, ENS, 75230 Paris, France
}

(Reçu le 29 juillet 1994 ; accepté le $1^{\text {er }}$ juin 1995)

\begin{abstract}
Résumé - Cette étude concerne les répartitions spatiales d'individus ligneux dans un « fourré tigré " (végétation tropicale en bandes), au nord-ouest du Burkina Faso $\left(14^{\circ} 00 \mathrm{~N}\right.$ et $2^{\circ} 30 \mathrm{~W}$ environ). Les données (comptages, mesures) ont été recueillies sur des transects de $700 \mathrm{~m}$ de long, orientés dans le sens de la pente, et divisés en placettes de $4 \mathrm{~m}^{2}$ et de $156 \mathrm{~m}^{2}$. Les répartitions spatiales ont été étudiées par calcul de variogrammes ; la structure d'ensemble du « fourré tigré " a été exprimée sous la forme d'un modèle qualitatif, dont l'efficacité à rendre compte des répartitions spatiales a été testée par régression linéaire sur variables « muettes ". Parmi les deux espèces dominantes, seul Combretum micranthum $\mathrm{G}$ Don présente des répartitions spatiales nettement liées à la structure d'ensemble. Celles de Pterocarpus lucens Lepr en sont plus affranchies, du fait d'une régénération par graines dynamique (extension sur les franges des bandes de végétation), en dehors des périodes de sécheresse. Le peuplement de $C$ micranthum ne présente pas d'évolutions nettes du fait d'une très faible survie des plantules au cours de la première saison sèche suivant la germination. En particulier, une progression vers l'amont de cette espèce, donc des fourrés, n'est pas actuellement perceptible.
\end{abstract}

Afrique occidentale / fourré tigré / structure de végétation / analyse des répartitions spatiales / évolution du couvert végétal

Summary - Structural analysis of a striped thicket in northern Yatenga (Burkina Faso): current state and dynamic insights. This study deals with spatial distributions of woody plants in a fourre tigré (tropical striped vegetational pattern) in northwestern Burkina Faso (ca $14^{\circ} 00^{\prime} \mathrm{N}, 2^{\circ} 30^{\prime} \mathrm{W}$ ). Data collection (censuses, measurements) was carried out on $700 \mathrm{~m}$ long transects, oriented along the slope, and divided into plots $\left(156 \mathrm{~m}^{2}\right)$ and quadrats $\left(4 \mathrm{~m}^{2}\right)$. Pattern analysis was based upon computations of variograms. The overall structure of the striped thickets was qualitatively modelled in order to test its influence on spatial distributions (using linear regression on 'dummy' variables). Among the two dominant species, only Combretum micranthum $G$ Don displayed spatial distributions clearly linked to the overall structure. Those of Pterocarpus lucens Lepr were comparatively more independent, due to their ability to expand on the fringes of vegetation stripes during periods of normal rainfall. The population of $\mathrm{C}$ micranthum did not display any clear trend because of a very low survival rate of seedlings during the first dry season following the germination. Hence, an upslope migration of the species is not perceptible at present.

Western Africa / striped vegetation / pattern analysis / vegetation change 


\section{INTRODUCTION}

Des couverts végétaux, fortement contrastés, sous forme d'une alternance de bandes couvertes de végétation et de bandes nues, ont été décrits, sous les noms de « brousse tigrée » ou de " fourré tigré », assez régulièrement dans la zone tropicale semi-aride, en Somalie (Boaler et Hodge, 1964), au Mexique (Montana et al, 1990) et en Afrique occidentale, depuis la Mauritanie jusqu'au Niger (Clos-Arceduc, 1956).

Ils apparaissent généralement sur des glacis à faible pente régulière ; les bandes de végétation étant grossièrement parallèles aux courbes de niveau. Très tôt des schémas d'interprétation à la fois fonctionnels et « dynamiques » ont été proposés par White (1970 et 1971), puis rediscutés par Greig-Smith (1979). Des études fines effectuées au Mexique (Cornet et al, 1988 ; Mauchamp et al, 1994) ont confirmé ces schémas, à savoir la redistribution de l'eau de la bande nue vers la bande de végétation, et le probable lent déplacement des bandes de végétation vers l'amont. Au Niger, Ambouta (1984) retient des hypothèses à peu près similaires.

Au nord Yatenga (nord-ouest du Burkina Faso), des fourrés tigrés peuvent aussi être observés ; ils représentent l'extrémité méridionale d'un ensemble plus vaste, s'étendant entre le $14^{e}$ et le $16^{\circ}$ parallèle (Boudet, 1972 ; Leprun, 1992). Mieux comprendre les propriétés et les tendances évolutives de ces formations passe obligatoirement par une analyse préalable de certains de leurs caractères structuraux; en particulier de ceux perceptibles dans le plan horizontal, là où s'exprime le plus l'hétérogénéité de ces formations.

Le but de ce travail n'est pas de démontrer l'existence d'une structure horizontale en bandes - à l'évidence, il y en a une, même si son intensité est variable dans l'espace. Il s'agit plutôt de cerner comment les principales espèces ligneuses constitutives exploitent la variabilité spatiale du couvert végétal ; à partir de là, d'explorer les implications de ces écologies différentes sur les cinétiques (nec « dynamiques ») probables des fourrés tigrés du nord Yatenga.

\section{MATÉRIEL ET MÉTHODES}

\section{Contexte écologique}

Situé à proximité de la frontière du Mali, le nord Yatenga s'étend approximativement entre $13^{\circ} 45$ et $14^{\circ} 20$ de latitude nord, d'une part, et $2^{\circ} 20$ et $2^{\circ} 45$ de longitude ouest, d'autre part. Une caractérisation d'ensemble du modelé et des sols peut être trouvée chez Guillet (1992).

Le climat est de type soudano-sahélien avec une saison des pluies de 3 à 4 mois (entre juin et septembre). Les principales caractéristiques climatiques ont été présentées par Serpantié et al (1992). Les précipitations ont connu des variations notables au cours des 50 dernières années soit :

- une période humide, depuis les années 1920 , avec une moyenne annuelle supérieure à $600 \mathrm{~mm}$;

- une période sèche, débutant en 1968 et connaissant son paroxysme en $1984(247 \mathrm{~mm})$, avec une moyenne d'à peine plus de $400 \mathrm{~mm}$.

Depuis 1986, on assiste à une remontée sensible du niveau de précipitations, avec une moyenne annuelle d'environ $525 \mathrm{~mm}$ par an ( $\sigma=90 \mathrm{~mm} / \mathrm{an}$ ).

Les années 1991 et 1992 ont connu des régimes de précipitations similaires avec 600 et $610 \mathrm{~mm}$ respectivement, assez bien réparties ; 1993 a connu un total comparable mais avec un fort retard du début de saison. En revanche, 1990 n'avait reçu que $370 \mathrm{~mm}$, avec une longue période de sécheresse en août (toutes ces valeurs correspondent aux données de l'Orstom pour la station de Bidi-Nayiri, $13^{\circ} 55 \mathrm{~N}$ et $2^{\circ} 30 \mathrm{~W}$ ).

\section{Les fourrés tigrés du nord Yatenga}

\section{Place dans le paysage}

Les fourrés tigrés n'occupent qu'une faible surface (moins de $10 \%$ des formations peu anthropisées) ; leur apparition est limitée à des situations morphologiques très particulières, à savoir des glacis réguliers, de pente comprise 
entre 0,5 et $1 \%$. Une pente régulière de cet ordre de grandeur paraît être un critère déterminant.

Dès lors que le modelé génère des situations de ce genre, l'apparition des fourrés tigrés est relativement indépendante de conditions de substratum géologique, puisque l'on peut en observer aussi bien sur granite que sur les grès argileux du «Continental terminal ".

Quelles que soient les conditions géologiques, les sols portant des fourrés tigrés sont fréquemment marqués par d'anciens cycles de cuirassement et sont généralement peu profonds $(30 \mathrm{~cm}$ à $1 \mathrm{~m}$ ), avec une forte charge de gravillons ferrugineux (débris de cuirasse) à faible profondeur. Sur granite, le substratum peut correspondre, par places, à une couverture d'altération épaisse et meuble (arène granitique).

Nulle part, l'alternance des bandes de végétation et des inter-bandes peu couvertes n'a pu être rattachée à des variations de conditions pédologiques; en revanche, la présence de la végétation occasionne de légères différences dans le fonctionnement hydrique du sol, avec des traces d'hydromorphie saisonnière, apparaissant un peu plus près de la surface, sous couvert (Ouedraogo, 1992 et données non publiées).

Les sols des glacis cuirassés sur lesquels se trouvent les formations tigrées ne sont presque jamais défrichés. L'usage pastoral actuel reste limité, du fait d'une végétation herbacée moins attractive que dans d'autres formations. Le ramassage de bois mort peut être important sur certains sites, mais il n'y a pas de coupes de bois vivant significatives.

\section{Physionomie}

Plusieurs parties (nommés par la suite « éléments ") peuvent être identifiées à l'intérieur d'une unité fonctionnelle, que nous nommerons " motif ", incluant la succession d'une bande de végétation et la surface peu couverte située immédiatement en amont ; soit (fig 1) :
- la surface peu couverte, avec des signes d'un fort ruissellement en nappe ; nous y distinguons une partie amont et une partie aval ;

- une frange à faible couvert ligneux, mais à fort couvert herbacé, présentant des signes nets d'arrêt et de décantation des eaux de ruissellement (" frange supérieure ") ;

- le fourré proprement dit, d'une hauteur moyenne de 2 à $4 \mathrm{~m}$, pouvant être surmonté par des arbres de plus de $5 \mathrm{~m}$;

- la « frange inférieure » du fourré, qui est, parfois, une limite relativement tranchée, mais aussi, souvent, une transition progressive. La végétation peut alors conserver un recouvrement encore notable dans la partie amont de la bande peu couverte du motif suivant, ce qui nous a amenés à opérer une distinction entre amont et aval.

Sur le terrain, ces éléments du motifs sont identifiables sur la base de la physionomie de la végétation, mais aussi de l'analyse des traces laissées par le ruissellement à la surface des sols ("croûtes ", Casenave et Valentin, 1989); lorsque l'aspect de la végétation n'est pas typique, ce sont ces indices du fonctionnement hydrique qui font foi pour l'identification. En effet, le schéma précédent est une vision idéalisée de la réalité (" un modèle $")$, plus ou moins reconnaissable selon les motifs. Ainsi, dans certains cas, une ou plusieurs des parties peuvent faire défaut (par exemple, il peut y avoir une bande herbeuse sans fourré vraiment constitué à l'aval, etc). Par endroits, la logique de motif peut même ne plus être perceptible à l'obsenvateur au sol. Même sur photographie aérienne (fig 2), l'identification n'est pas toujours nette.

Les fourrés tigrés sont dominés par Combretum micranthum (tableau I), un arbuste essentiellement multicaule et ne dépassant que rarement $5 \mathrm{~m}$ de haut, et Pterocarpus lucens, dont le port, variable selon les situations, peut être arbustif ou arboré. Ces deux espèces dominent d'ailleurs la plupart des formations végétales

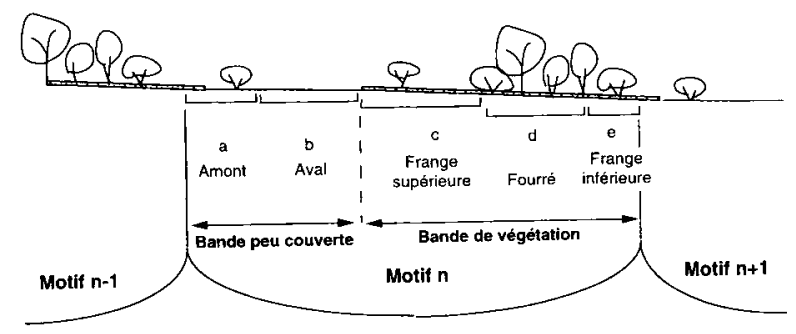

Fig 1. Modèle structurel d'un fourré tigré. 


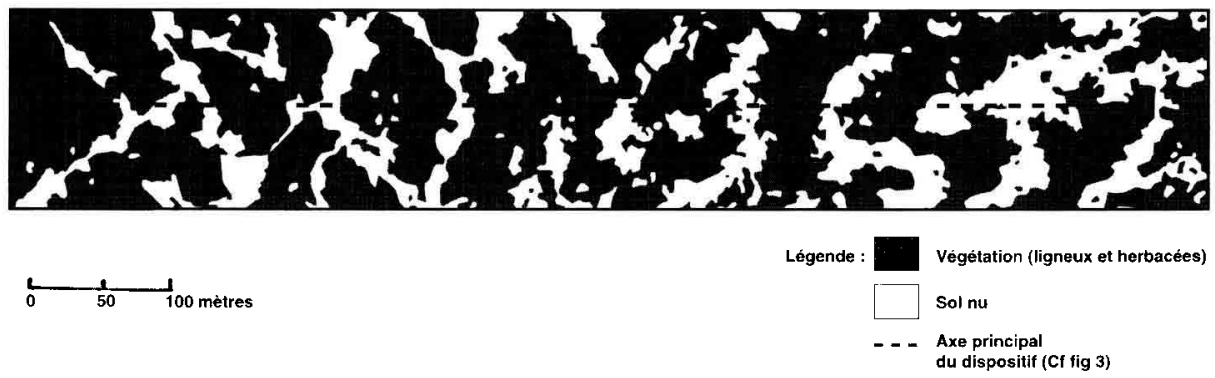

Fig 2. Cartographie du couvert végétal pour la portion de fourré tigré étudiée, obtenue par interprétation de photographies aériennes en couleur, prises en octobre 1994 (objectif $35 \mathrm{~mm}$, prise de vue à $1000 \mathrm{~m}$ de hauteur).

peu anthropisées de la région. La strate herbacée, discontinue, est principalement constituée de graminées annuelles; son développement et sa composition floristique sont variables selon les précipitations de l'année considérée.

\section{Méthodes}

\section{Inventaire des peuplements}

Une portion de fourré tigré bien structuré a été sélectionnée sur un glacis peu incliné, à environ $4 \mathrm{~km}$ de la station de Bidi. Ce glacis, d'environ $3 \mathrm{~km}$ de long et de $2 \mathrm{~km}$ de large, relie un sommet cuirassé à un bas-fond. Seule la moitié supérieure du glacis porte un fourré tigré bien structuré sur une distance d'environ $1 \mathrm{~km}$ dans le sens de la pente. Celle-ci est d'environ 0,7\% pour l'ensemble du transect (Ouedraogo, 1992) ; le substrat est granitique.

L'échantillonnage retenu se base sur une maille principale composée de 224 grandes placettes de $12,5 \times 12,5 \mathrm{~m}$ (fig 3 ), soit 3,4 ha. L'orientation du dispositif (fig 2) est conforme avec la pente générale du glacis (mais pas forcément partout avec la pente locale). Pour décrire plus finement la structure horizontale des fourrés tigrés, deux transects composés de petites placettes contiguës de $4 \mathrm{~m}^{2}(2 \times 2 \mathrm{~m})$ ont été disposés à l'intérieur du dispositif principal.

Tous les individus ligneux rencontrés sur les petites placettes sont pris en compte et mesurés (hauteur totale, circonférence au ras du sol, circonférence à $40 \mathrm{~cm}$ du sol de la plus grosse tige, ainsi que le plus grand diamètre de la couronne et son diamètre perpendiculaire). La distinction entre " semis" de l'année et individus d'au moins un an a été réalisée sur la base de la présence des feuilles cotylédonaires (généralement visibles jusqu'à la fin septembre).

En ce qui concerne les grandes placettes, les " jeunes " individus (hauteur $<50 \mathrm{~cm}$ ), et tous les $C$ micranthum, déjà nombreux sur les petites placettes, ne sont plus mesurés ; en revanche, tous les autres individus ligneux sont pris en

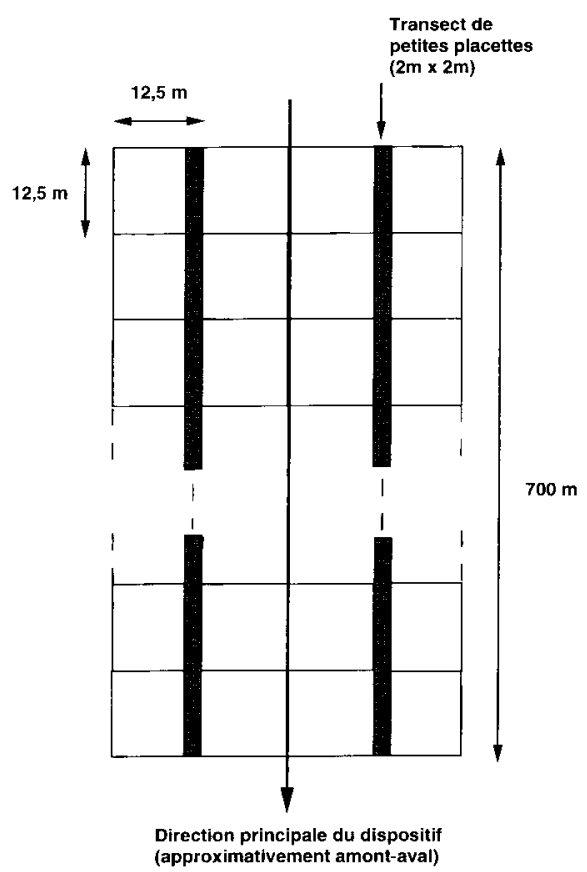

Fig 3. Dispositif d'échantillonnage. 
compte avec mesure des mêmes variables que précédemment.

Même si d'autres variables ont été considérées (surfaces terrières, indices de couvert), les traitements effectués ci-après s'appuient essentiellement sur des effectifs par catégorie d'individus (espèces, classes de hauteur, de grosseur). En particulier, sera retenu le découpage, basé sur la hauteur totale $(H)$, en " jeunes " $(\mathrm{H}<50 \mathrm{~cm})$, " sub-adultes " $(50 \leq \mathrm{H}<150 \mathrm{~cm})$ et « adultes" $(\mathrm{H} \geq 150 \mathrm{~cm})$. Les dénominations sont, bien sûr, approximatives; elles semblent cependant bien correspondre au cas de $P$ lucens. Pour les espèces buissonnantes, les deux dernières catégories sont fréquemment fusionnées dans les traitements.

L'inventaire des individus morts et encore sur pied a été réalisé ; l'interprétation des résultats est néanmoins délicate, compte tenu du fait que, sous les conditions pluviométriques actuelles, le taux annuel de mortalité est faible, et ne peut pas être appréhendé efficacement par un suivi sur 2 ou 3 ans. II faut donc tenter d'interpréter le nombre d'individus morts, comptés en 1992, qui représente un cumul, sur plusieurs années, d'une mortalité annuelle. Cette approche ne peut être envisagée que pour les adultes des deux espèces dominantes, les autres étant trop peu fréquentes. Elle demande des informations sur la durée de conservation d'un individu mort.

En ce qui concerne $P$ lucens, les adultes morts sont facilement repérables pendant plus d'une décennie ; ainsi, ceux, victimes de la période la plus sèche du siècle (de 1981 à 1984), nombreux à $40 \mathrm{~km}$ au nord du site d'étude (Couteron et al, 1992), sont encore presque tous sur pied ; les individus morts comptés en 1992 incluent donc ceux de la période de crise. Pour $C$ micranthum, les individus morts ne se conservent pas aussi longtemps, et ceux morts au début des années 1980 ont fréquemment disparu, bien que leurs ensouchements (" chicots "), puissent encore être repérés avec un peu d'attention. II est donc raisonnable de penser que les individus, morts et encore debout, comptés en 1992 traduisent essentiellement la mortalité de la période 1985-1992, sous des précipitations " normales ".

Deux grands types d'analyses statistiques ont été envisagés :

- celles permettant d'acquérir des informations sur les répartitions spatiales d'individus, ou de classes d'individus, que nous dénommons par la suite analyses des répartitions spatiales ;
- celles permettant de décrire les variations de variables de peuplement par rapport à la structure d'ensemble du fourré tigré (analyse physionomique). Les deux approches sont bien évidemment complémentaires : les résultats de la première ont le mérite de ne pas imposer l'usage d'un modèle structurel défini a priori ; leur interprétation écologique peut néanmoins être délicate (Grace, 1989). Cette interprétation peut alors être étayée par les résultats de la seconde approche.

\section{Analyses des répartitions spatiales}

Les dispositifs composés de placettes contiguës ou emboîtées se prêtent bien à l'analyse des répartitions spatiales d'individus végétaux (Greig-Smith, 1979 ; Grace, 1989). Plusieurs méthodes de traitement sont envisageables (Chessel, 1978 ; Cressie, 1993). Parmi celles-ci nous retenons principalement l'analyse de la différence entre placettes en fonction de leur distance. Cette dernière a été réalisée par calcul de variogrammes (Matheron, 1970 ; Cressie, 1993 p 58), technique ayant déjà fait l'objet de nombreuses applications fructueuses, concernant la structure horizontale de peuplements ligneux (par exemple, Bachacou et Decourt, 1976 ; Bariteau, 1992). Le variogramme expérimental, $\gamma(h)$ est calculé par :

$$
\gamma(h)=\frac{1}{2 N} \sum_{i=1}^{N}(x i+h-x i)^{2}
$$

où les $x i$ sont les observations, sur $n$ placettes, de la variable étudiée, et où $h$ est la distance entre deux placettes, définie, selon la direction principale du dispositif (fig 3). $N$ est le nombre de couples que l'on a pu former à partir des $n$ placettes initiales. Le variogramme permet une description des structures spatiales, moyennant une seule hypothèse sous-jacente, "l'hypothèse intrinsèque " de stationnarité d'ordre 2 des accroissements de la variable étudiée ; il est en cela d'application plus large que le corrélogramme, méthode d'analyse étroitement apparentée.

\section{Analyses physionomiques}

Le schéma de structure d'un motif du fourré tigré, proposé à la figure 1, correspond à une variable concomitante qualitative en six modalités (les cinq définies à la figure 1 plus une sixième codant éventuellement les segments difficiles à interpréter). Pour évaluer l'efficacité de ce modèle à rendre compte de la variabilité de la couverture végétale ligneuse, le modèle linéaire suivant 
(noté, A) a été ajusté, par la méthode des moindres carrés, pour chaque variable $Y$ de peuplement :

$$
y=\sum_{i=1}^{5} \text { aixi+e }
$$

Ceci correspond à un modèle de régression linéaire sur variables " muettes " (dummy variables), où $y$ est l'observation de la variable de peuplement. Les $x i$ sont les valeurs prises par des variables booléennes, $X i$, correspondant chacune à une des modalités de la variable qualitative concomitante $(x i=1$ si l'observation considérée est caractérisée par la ième modalité de la variable et $x i=0$, sinon). En pratique on ne retient que cinq variables $X i$, puisque $\Sigma x i=1$, quelle que soit l'observation prise en compte; les termes ai sont les coefficients de la régression, et e est le résidu d'ajustement. Le coefficient de détermination de la régression, $\eta^{2}(Y, A)$, mesure l'influence de la variable qualitative $A$ (structure interne du motif) sur la variable de peuplement $Y$. Il sera utilisé de façon descriptive pour comparer les influences de $A$ sur plusieurs variables de peuplement.

Une autre source de variation des variables de peuplement peut être supposée résulter des différences entre motifs; celle-ci est prise en compte, comme une nouvelle variable qualitative $B$, dans un modèle plus complet :

$$
y=\sum_{i=1}^{5} a i x i+\sum_{j} b j z j+e
$$

où les $Z$ j sont des variables qualitatives booléennes, codant l'appartenance aux différents motifs rencontrés par le dispositif, et les bj les coefficients leur correspondant. Ce modèle est noté « $A+B$ »; son coefficient de détermination, $\eta^{2}(Y, A+B)$, exprime l'influence, sur la variable de peuplement $Y$, des variables qualitatives $A$ et $B$, prises conjointement. La différence $\eta^{2}(Y$, $A+B)-\eta^{2}(Y, A)$ mesure l'influence de $B$, sachant que celle de $A$ a déjà été prise en compte ( $B / A »$ ). Ceci permet d'apprécier la variabilité entre les motifs, indépendamment de la structure interne au motif.

L'analyse peut être poussée plus loin en créant un nouvel ensemble de variables booléennes, $T K$, codant la combinatoire des variables $X i$ et $Z j$ (les $T k$ sont appelés " tableau d'interaction " et correspondent à une nouvelle variable qualitative noté « $A B »$ ). Un nouveau modèle (noté « $A+B+A B »)$ peut s'écrire :

$$
y=\sum_{i=1}^{5} a i x i+\sum_{j} b j z j+\sum_{k} c k t k+e
$$

$\eta^{2}(Y, A+B+A B)-\eta^{2}(Y, A+B)$ permet de mesurer l'influence de $A B$ sur $Y$.

L'ensemble des calculs a été effectué avec le logiciel Biomeco (Lebreton et al, 1990). Les modèles précédents ont été utilisés pour les deux tailles de placettes. La transformation de variable $y=\sqrt{x}$ a été systématiquement réalisée sur les variables de comptage; elle est adaptée à la normalisation et à l'homogénéisation des variances pour des variables de Poisson.

Au-delà de l'évaluation d'effets globaux, l'analyse des coefficients des régressions permet une approche plus détaillée. Les coefficients ai et $b j$ correspondent aux moyennes de la variable $Y$ pour les modalités des variables explicatives (après transformation par $y=\sqrt{x}$ ). En complément, la proportion de placettes occupées par une catégorie d'individus (logique de présence/absence) a aussi été prise en considération. A effectifs comparables, un taux de placettes occupées plus fort traduira une répartition moins agrégative. Les variations de la proportion de placettes occupées, selon les éléments structuraux d'un motif, peuvent être testée par le " test exact de probabilité de Fisher » (Siegel, 1956 p 106).

\section{Suivi des jeunes individus}

En plus des comptages effectués en 1992 sur le dispositif principal, 27 placettes de $5 \times 5 \mathrm{~m}$ ont été implantées en 1993, juste avant les pluies (juin), dans trois bandes de végétation choisies aléatoirement. Pour chaque bande, trois placettes sont situées dans le fourré et autant dans chacune de ses franges. Les jeunes individus présents (hauteur $<50 \mathrm{~cm}$ ) ont été bagués ; les placettes ont ensuite été visitées régulièrement avec relevé des individus disparus et baguage des plantules nouvellement levées. Ce dispositif a permis d'estimer les taux de mortalité des jeunes individus sur le cycle annuel 1993-94.

\section{RÉSULTATS}

\section{Résultats généraux}

À partir des résultats obtenus sur les transects de $2 \mathrm{~m}$ de large, on relève une importance relative de :

- $25 \%$ pour les franges supérieures (ce chiffre important témoigne du fort dévelop- 
pement du couvert herbeux, depuis la fin de la période sèche);

$-20 \%$ pour les fourrés ;

$-8 \%$ pour les franges inférieures ;

- $15 \%$ pour la partie amont des bandes peu couvertes ;

$-20 \%$ pour la partie aval des bandes peu couvertes.

Le reste (12\%) est constitué par des placettes difficiles à imputer à l'une de ces catégories (en relation avec des bandes ou des motifs très peu structurés). D'après ces chiffres, les bandes de végétation représentent $53 \%$ de la surface. Cette proportion doit néanmoins être augmentée pour tenir compte du fait que la plupart des placettes difficiles à imputer sont végétalisées ; de plus, les bandes peu couvertes ne sont pas entièrement nues. Le couvert végétal est donc plus étendu que les sols nus dans une proportion d'environ deux tiers à un tiers (cf aussi fig 2).

Le peuplement adulte représente à peu près 550 individus/ha, pour une surface terrière de $3,1 \mathrm{~m}^{2} / \mathrm{ha}$. Cette dernière est constituée principalement par $C$ micranthum (57\% du total) et $P$ lucens ( $28 \%$ ). Les autres espèces ligneuses (11 recensées) n'ont donc qu'une importance très secondaire (15\%). Il s'agit de Balanites aegyptiaca, Boscia senegalensis, $B$ salicifolia, Cadaba farinosa, Combretum nigricans, Gardenia sokotensis, Grewia bicolor, G flavescens, Guiera senegalensis, Lan- nea acida, Piliostigma reticulatum. Les sept espèces les plus fréquemment rencontrées sont présentées au tableau I.

Les adultes de $P$ lucens, soit 43 individus/ha, en moyenne (densité sur les grandes placettes allant jusqu'à 320/ha) ont une hauteur moyenne de 4,2 $\mathrm{m}$ $(\sigma=2 \mathrm{~m})$, et une surface moyenne de couronne de $16 \mathrm{~m}^{2}$. A peu près $32 \%$ des individus adultes ont une hauteur supérieure à $5 \mathrm{~m}$, avec, dans ce cas, une surface moyenne de couronne de $33 \mathrm{~m}^{2}$. P lucens représente l'essentiel du couvert supérieur à $5 \mathrm{~m}$ (et $95 \%$ des individus supérieurs à ce seuil).

Les adultes de $C$ micranthum représentent 385 et 405 individus/ha, selon les deux transects, respectivement. Leur hauteur moyenne est de $2,6 \mathrm{~m}(\sigma=0,6 \mathrm{~m})$, pour une surface de couronne moyenne de $4,5 \mathrm{~m}^{2}$. Aucun individu supérieur à $5 \mathrm{~m}$ n'a été rencontré.

Le nombre d'individus morts est très faible pour Pterocarpus lucens $(1,5 \%$ du nombre d'individus comptés) ; ceci signifie que non seulement la mortalité annuelle actuelle est extrêmement faible, mais aussi que la dernière sécheresse (1968-85) a très peu affecté ce peuplement, ce qui n'est pas le cas d'autres sites, un peu plus au nord (Couteron et al, 1992). En ce qui concerne $C$ micranthum, la proportion d'individus morts, encore sur pied, est plus élevée (11\%).

Tableau I. Présentation des principales espèces ligneuses. Les sept espèces présentées sont les plus abondantes dans les comptages et sont les seules mentionnées dans la suite du texte.

\begin{tabular}{llll}
\hline Nom scientifique & Nom peul & Famille & Type biologique \\
\hline Acacia ataxacantha DC & & & \\
Combretum micranthum G Don & noorahi & Mimosaceae & Buisson, liane \\
Gardenia sokotensis Hutch & koolel ferro & Combretaceae & Arbuste \\
Grewia flavescens Juss & ciibooli & Tilliaceae & Buisson \\
Grewia bicolor Juss & kelli & Tilliaceae & Buisson \\
Guiera senegalensis J F Gmelin & ngelooki & Combretaceae & Arbuste \\
Pterocarpus lucens Lepr & cami & Fabaceae & Arbuste / arbre
\end{tabular}


La densité des jeunes individus est importante pour l'ensemble du peuplement (près de 10000 jeunes individus vivants/ha); les deux espèces principales contribuent le plus fortement à la régénération $(90 \%$ des jeunes individus). Malgré une forte variabilité spatiale, les jeunes $P$ lucens sont répartis sur une surface considérable : $44 \%$ des petites placettes en contiennent au moins un $\left(1,83\right.$ jeune $\left./ 4 \mathrm{~m}^{2}\right)$; ce ratio est légèrement plus faible pour $C$ micranthum ( $40 \% ; 1,57$ jeune par petite placette).

\section{Répartitions spatiales}

Sur les petites placettes de $4 \mathrm{~m}^{2}$, plusieurs formes de variogrammes peuvent être observées (fig 4). Dans la plupart des cas, la discontinuité à l'origine (" effet de pépite ») est forte (plus de $50 \%$ des valeurs maximales atteintes), dénotant une forte hétérogénéité entre placettes adjacentes. Audelà, le variogramme peut présenter une croissance rapide (cas du $C$ micranthum, pour toutes les classes d'âge) ou modérée (jeunes $P$ lucens).

Plusieurs variogrammes ont une allure périodique, avec des extrema locaux bien individualisés ; ceci est particulièrement vrai pour les jeunes et les adultes de $C \mathrm{mi}$ cranthum, même si, dans le deuxième cas, la forme n'est pas aussi nette sur les deux transects. Cette forme reproduit assez fidèlement l'alternance des arcs de végétation et des bandes peu couvertes, avec, un premier pic vers $40 \mathrm{~m}$, un creux vers $70 \mathrm{~m}$ et un palier atteint vers $110 \mathrm{~m}$. Ces chiffres sont à rapprocher de la taille moyenne des motifs qui est d'environ $60 \mathrm{~m}(\sigma=25 \mathrm{~m})$, d'après calculs sur photographies aériennes. Pour les sub-adultes de $C$ micranthum, la forme du variogramme est différente, et correspond assez bien à un “ modèle sphérique ».

Pour les jeunes $P$ lucens, une périodicité plus discrète peut aussi être relevée (fig 4) ; elle est cependant relativement dé- phasée par rapport à la précédente, avec, un premier pic plutôt vers $10 \mathrm{~m}$.

Pour les grandes placettes de $156 \mathrm{~m}^{2}$ (fig 4), la discontinuité à l'origine reste forte ; le comportement périodique n'apparaît que pour une espèce (Gardenia sokotensis), effectivement inféodée aux fourrés (sensu stricto). Pour les autres espèces, les variogrammes sont relativement plats, dénotant plutôt une certaine absence de structure; ceci, à l'exception des $P$ /ucens sub-adultes dont la première partie du variogramme présente une certaine similitude avec un modèle sphérique (avec une «portée » d'environ $50 \mathrm{~m}$ ). Sur des distances plus longues, ce même variogramme comporte un fléchissement qui semble significatif, car il concerne huit points consécutifs du graphe, tous calculés sur plus de 100 couples de placettes.

Ces analyses peuvent être complétées par l'examen des cartographies de la figure 5. Conformément au variogramme, la répartition des Boscia senegalensis (adultes et sub-adultes) ne laisse pas apparaître de structure très marquée (si ce n'est des densités plus faibles à l'aval du dispositif). Guiera senegalensis est, en revanche, plus dense dans la partie aval du dispositif, à l'approche des jachères du bas de glacis ; dans la partie amont, les individus sont trop dispersés pour que l'analyse visuelle puisse identifier une structure particulière. Pour les Pterocarpus lucens subadultes, la principale structure est l'existence de 2 ensembles plus denses, d'un peu plus de $100 \mathrm{~m}$ de long, et séparés par une portion moins dense d'un peu plus de $200 \mathrm{~m}$; c'est cette structure qui marque le variogramme. Pour les adultes de la même espèce, il n'y a pas de structure bien nette, à l'exception d'une petite plage plus dense à l'extrémité aval. En revanche, pour G sokotensis, apparaît une certaine structure en bandes, globalement perpendiculaires à l'axe du dispositif.

Pour les autres espèces, une telle structure n'apparaît pas vraiment, soit parce que 
a) Petites placettes $(4 \mathrm{~m} 2)$
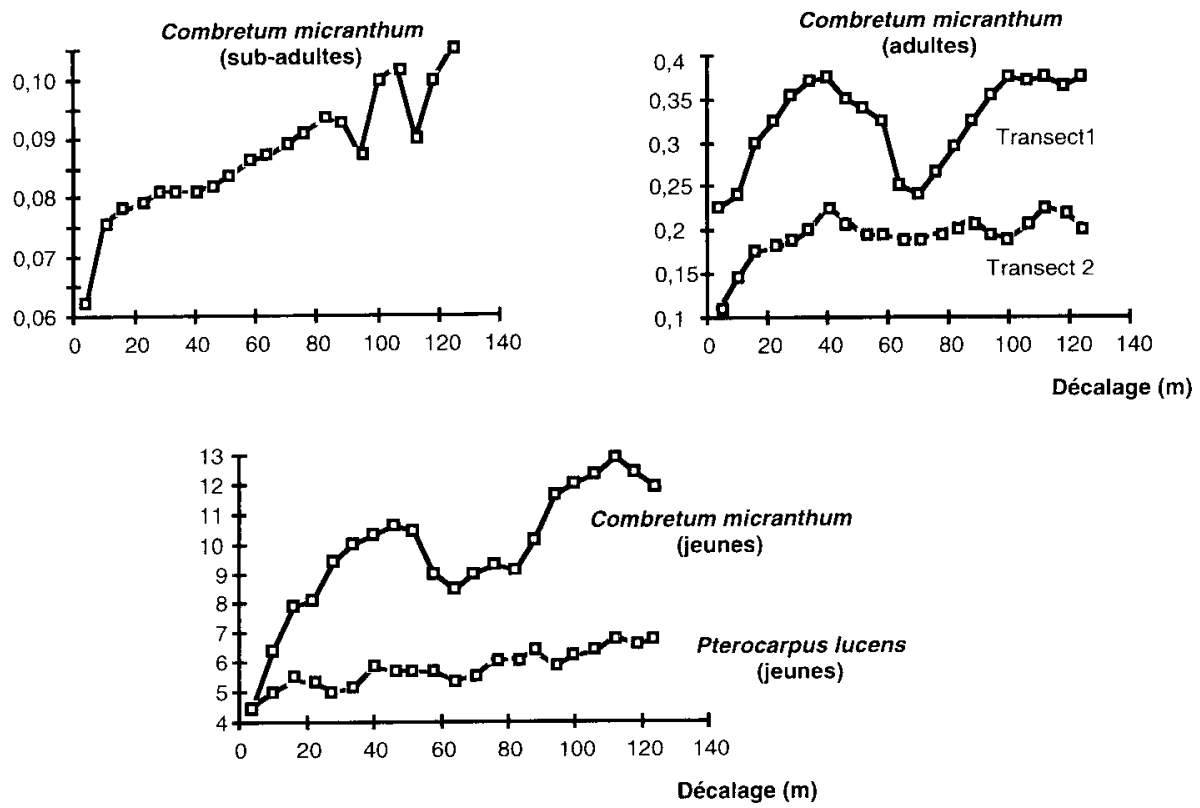

b) Grandes placettes (156 m2)
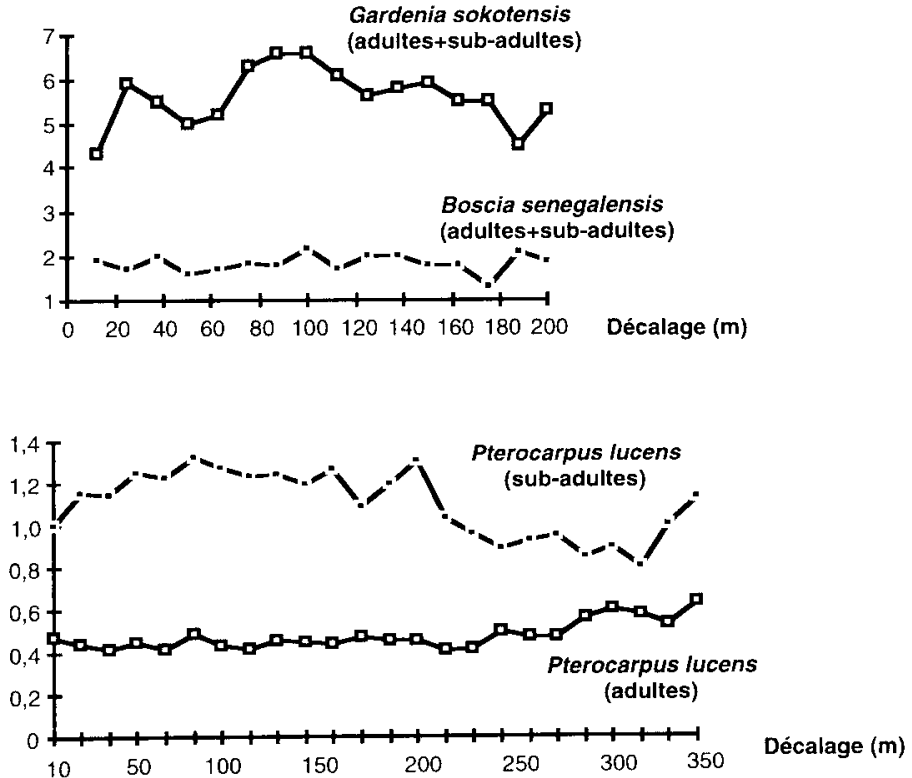

Fig 4. Variogrammes expérimentaux. 
les individus sont trop peu nombreux et dispersés, soit parce que certains d'entre eux sont néanmoins localisés dans les bandes peu couvertes (le cas le plus net étant celui de Boscia senegalensis).

\section{Analyses physionomiques}

La figure 6 synthétise le pouvoir explicatif des différents modèles pour quelques catégories d'individus.

\section{Influence de la structure du motif (modèle A)}

Cette influence est plus ou moins importante selon les variables considérées. Pour les petites placettes, les variables les mieux expliquées sont, fort logiquement, celle correspondant aux effectifs du peuplement adulte (densités, indices de couvert, etc) de $C$ micranthum, avec des coefficients de détermination entre 0,2 et 0,35 (selon les transects). C'est cette catégorie d'individu qui crée la structure d'ensemble, et il est normal que sa variabilité soit assez bien expliquée par ce premier modèle. L'effectif de jeunes individus (toutes espèces confondues) est aussi assez bien expliqué, avec un coefficient de détermination de 0,25; ce coefficient est de 0,14 pour $C$ micranthum et de 0,11 pour $P$ lucens. Dans l'ensemble, le pouvoir explicatif est faible en ce qui concerne les effectifs de jeunes individus des espèces secondaires (avec des coefficients de 0,05 à 0,07).

Sur les grandes placettes, le meilleur pouvoir explicatif s'obtient pour G sokotensis (adultes et sub-adultes confondus), avec un coefficient de détermination de 0,20. Les coefficients sont nettement plus faibles pour les autres espèces : 0,07 et 0,06 pour les adultes de $P$ lucens et Guiera senegalensis, respectivement ; et seulement 0,02 pour Boscia senegalensis (adultes et sub-adultes confondus), dont la répartition se confirme bien être relativement indépendante de la structure interne des motifs.

Le tableau II donne les valeurs moyennes des effectifs en fonction des éléments d'un motif. Pour les deux espèces dominantes,
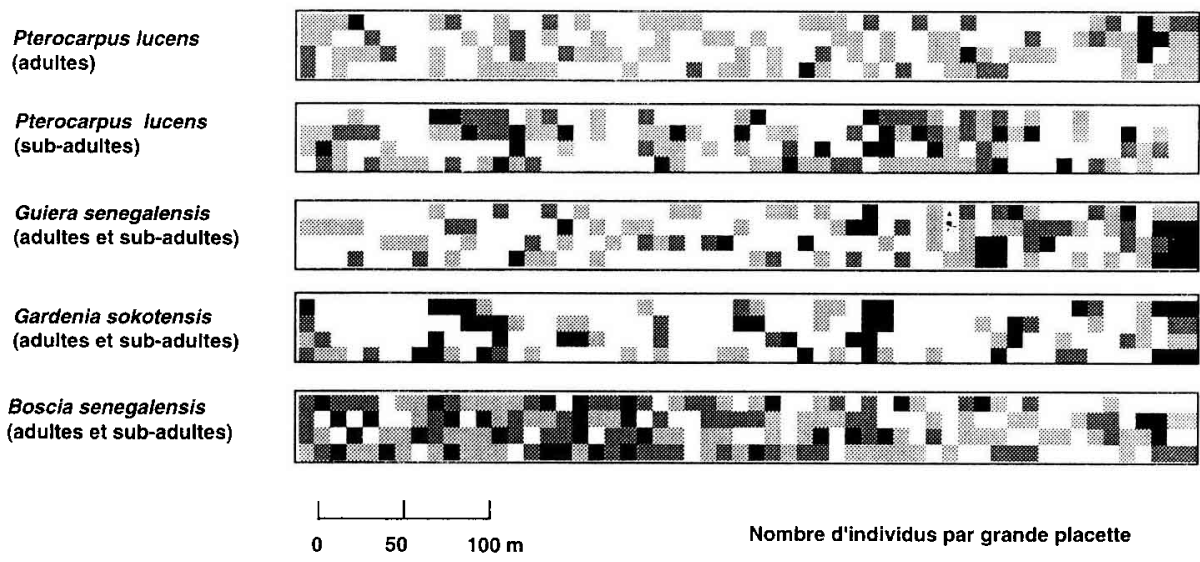

Nombre d'individus par grande placette

Sens de la pente

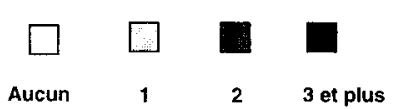

Fig 5. Cartographie des effectifs observés sur les grandes placettes $\left(156 \mathrm{~m}^{2}\right)$, pour cinq catégories d'individus. 
les jeunes individus sont nombreux dans toute la bande de végétation, ainsi que dans la partie amont des bandes peu couvertes. Les jeunes $P$ lucens sont particulièrement nombreux dans les franges supérieures des bandes de végétation; la répartition n'est pas sensiblement différente pour les semis de l'année et pour les jeunes de plus d'un an. La densité des subadultes de $P$ lucens est peu variable à l'intérieur d'une bande de végétation ; elle reste notable dans le haut des bandes peu couvertes. La densité de $B$ senegalensis décroît peu dans les bandes peu couvertes. En revanche, $G$ sokotensis est très lié au fourré et à la frange supérieure. De même, G senegalensis est plus dense dans les franges supérieures que partout ailleurs.

L'analyse des taux de placettes occupées permet de porter un regard complémentaire sur les répartitions à l'intérieur du motif (tableau III). Dans l'ensemble, ces taux varient de façon conforme aux effectifs. Quelques exceptions apparaissent en ce qui concerne les sub-adultes des deux espèces dominantes. Pour $\mathrm{C}$ micranthum, les variations d'effectifs, selon les éléments du motif, ne se traduisent que par peu de différences significatives dans les taux d'occupation ; ceux-ci sont globalement faibles et peu variables. Pour $P$ lucens, les franges supérieures se caractérisent, à effectifs équivalents avec les franges infé-

a) Grandes placettes (156 m2)
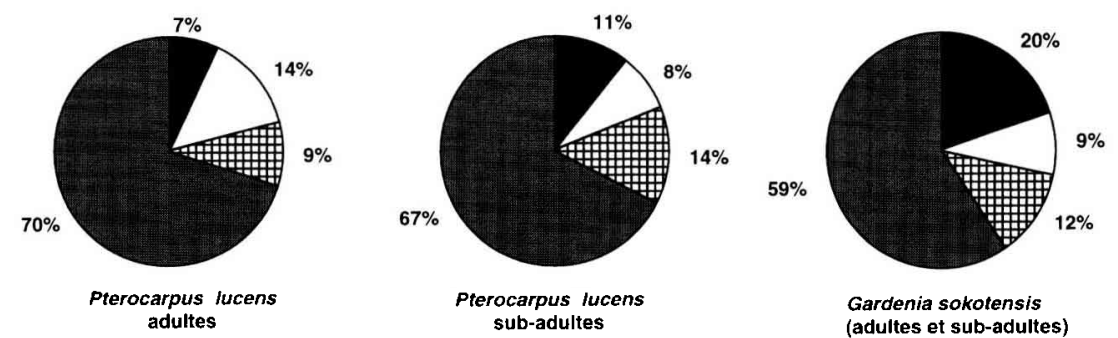

b) Petites placettes ( $4 \mathrm{~m} 2$ )
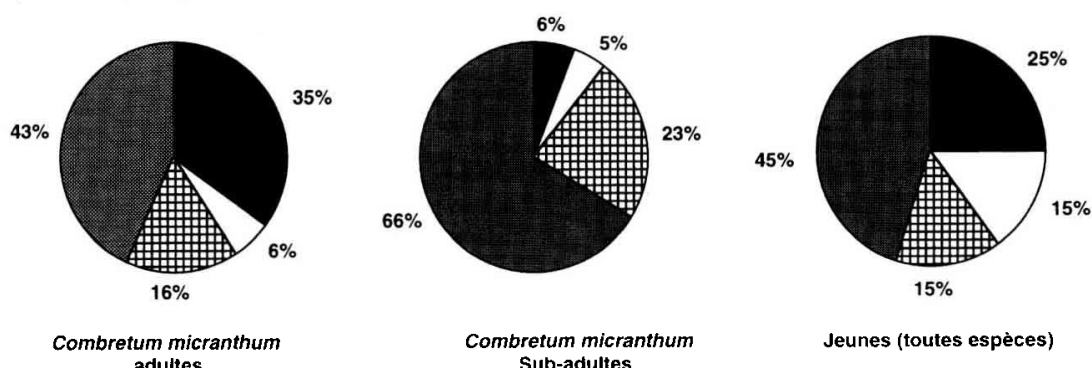

Combretum micranthum Sub-adultes

Jeunes (toutes espèces)

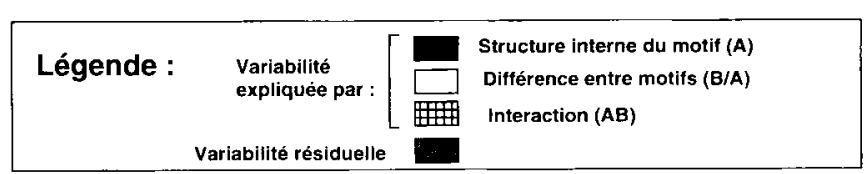

Fig 6. Décomposition de la variabilité selon les modèles, pour six catégories d'individus. 
Tableau II. Effectifs selon les éléments d'un motif (les effectifs sont ramenés à l'hectare, pour les deux tailles de placettes de comptage).

\begin{tabular}{|c|c|c|c|c|c|c|}
\hline Eléments du motif & & $\begin{array}{c}\text { Bande peu } \\
\text { couverte } \\
\text { (haut) }\end{array}$ & $\begin{array}{c}\text { Bande peu } \\
\text { couverte } \\
\text { (bas) }\end{array}$ & $\begin{array}{c}\text { Frange } \\
\text { supérieure }\end{array}$ & Fourré & $\begin{array}{c}\text { Frange } \\
\text { inférieure }\end{array}$ \\
\hline \multirow{2}{*}{$\begin{array}{l}\text { Boscia senegalensis } \\
\text { (adultes et sub-adultes) }\end{array}$} & Moyenne & 61,7 & 80,0 & 92,1 & 89,9 & 81,5 \\
\hline & $\mathrm{cV}$ & 1,09 & 0,77 & 1,03 & 0,84 & 0,93 \\
\hline \multirow{2}{*}{$\begin{array}{l}\text { Combretum micranthum } \\
\text { (jeunes) }\end{array}$} & Moyenne & 2125 & 550 & 5750 & 5750 & 4500 \\
\hline & $\mathrm{CV}$ & 2,35 & 4 & 1,78 & 1,61 & 1,61 \\
\hline \multirow{2}{*}{$\begin{array}{l}\text { Combretum micranthum } \\
\text { (sub-adultes) }\end{array}$} & Moyenne & 25 & 50 & 75 & 250 & 375 \\
\hline & $\mathrm{CV}$ & 12 & 6 & 7 & 3,6 & 2,33 \\
\hline \multirow{2}{*}{$\begin{array}{l}\text { Combretum micranthum } \\
\text { (adultes) }\end{array}$} & Moyenne & 88 & 44 & 73 & 1181 & 788 \\
\hline & $\mathrm{CV}$ & 3,8 & 5,7 & 5 & 1,15 & 1,44 \\
\hline \multirow{2}{*}{$\begin{array}{l}\text { Gardenia sokotensis } \\
\text { (adultes et sub-adultes) }\end{array}$} & Moyenne & 6,9 & 5,3 & 107,8 & 151,2 & 21,3 \\
\hline & $\mathrm{CV}$ & 2,89 & 4,80 & 1,52 & 1,19 & 1,76 \\
\hline \multirow{2}{*}{$\begin{array}{l}\text { Guiera senegalensis } \\
\text { (adultes et sub-adultes) }\end{array}$} & Moyenne & 32,0 & 13,3 & 97,7 & 47,7 & 42,7 \\
\hline & $\mathrm{CV}$ & 1,36 & 2,39 & 1,37 & 1,70 & 1,64 \\
\hline \multirow{2}{*}{$\begin{array}{l}\text { Pterocarpus lucens } \\
\text { (jeunes de l'année) }\end{array}$} & Moyenne & 2750 & 775 & 5000 & 2750 & 2750 \\
\hline & $C V$ & 2,0 & 4,19 & 2,60 & 2,55 & 1,46 \\
\hline \multirow{2}{*}{$\begin{array}{l}\text { Pterocarpus lucens } \\
\text { (jeunes d'au moins un an) }\end{array}$} & Moyenne & 1250 & 525 & 2125 & 1025 & 1750 \\
\hline & $\mathrm{CV}$ & 2,0 & 4,19 & 1,85 & 1,83 & 1,43 \\
\hline \multirow{2}{*}{$\begin{array}{l}\text { Pterocarpus lucens } \\
\text { (sub-adultes) }\end{array}$} & Moyenne & 29,4 & 2,6 & 81,9 & 80,6 & 65,9 \\
\hline & $\mathrm{cV}$ & 1,59 & 5 & 0,99 & 1,17 & 1,27 \\
\hline \multirow{2}{*}{$\begin{array}{l}\text { Pterocarpus lucens } \\
\text { (adultes) }\end{array}$} & Moyenne & 13,4 & 18,6 & 51,8 & 48,0 & 46,7 \\
\hline & $\mathrm{CV}$ & 2,33 & 2,1 & 1,15 & 1,24 & 1,03 \\
\hline
\end{tabular}

rieures, par un taux d'occupation nettement plus fort, ce qui traduit un peuplement mieux réparti. À noter aussi que le taux d'occupation n'est pas significativement moins fort dans le haut des bandes peu couvertes que dans le fourré. Les forts effectifs de jeunes Pterocarpus dans les franges supérieures ne se traduisent pas par un taux d'occupation plus fort que dans les franges inférieures (au contraire).

\section{Variabilité entre les motifs (modèle B/A)}

Elle est forte en ce qui concerne les effectifs des jeunes individus des deux espèces principales (coefficient de détermination de 0,27 ). Ceci est particulièrement net pour $C$ micranthum $(0,38)$ et pour les semis de l'année de $P$ lucens $(0,27)$, mais moins pour les jeunes Pterocarpus de plus d'un an. Les autres variables, relatives aux espèces principales ne sont que médiocrement expliquées par la variabilité entre les motifs (coefficients de détermination del'ordre de 0,05$)$. Ce chiffre est néanmoins un peu plus fort en ce qui concerne les adultes de $C$ micranthum $(0,09)$. Une traduction spatiale de cette variabilité entre motifs peut être obtenue en représentant les coefficients bj du modèle $A+B$, en fonction de 
la position du motif, le long de l'axe principal du dispositif. Pour $P$ lucens (non illustré), apparaît alors surtout le rôle de deux motifs exceptionnels, situés dans le dernier tiers du deuxième transect. Pour $C$ micranthum, l'un de ces deux motifs estaussi remarquable du point de vue des jeunes individus ; en revanche, l'effectif des adultes (fig 7) correspond à des logiques différentes : d'une part, une légère tendance à l'augmentation en descendant sur la toposéquence, mais surtout de fortes variations de densité entre motifs successifs. Les allures des graphiques obtenus, pour chacun des deux transects pris séparément sont, de plus, relativement concordantes, même si c'est le premier transect qui semble présenter la périodicité la plus nette.

\section{Interaction (modèle $A B$ )}

L'interaction représente une part importante de la variabilité de certaines variables : le coefficient de détermination du modèle $A B$ est, en moyenne, de l'ordre de 0,17 . Néanmoins, ce chiffre doit être relativisé du fait du nombre important de

Tableau III. Proportion de placettes occupées selon les éléments structuraux d'un motif. Traitement en présence/absence par le test exact de probabilité de Fisher (Siegel,1956, p 106).

\begin{tabular}{|c|c|c|c|c|c|}
\hline Eléments du motif & $\begin{array}{l}\text { Bande peu } \\
\text { couverte } \\
\text { (haut) }\end{array}$ & $\begin{array}{l}\text { Bande peu } \\
\text { couverte } \\
\text { (bas) }\end{array}$ & $\begin{array}{l}\text { Frange } \\
\text { supérieure }\end{array}$ & Fourré & $\begin{array}{l}\text { Frange } \\
\text { inférieure }\end{array}$ \\
\hline $\begin{array}{l}\text { Combretum micranthum } \\
\text { (jeunes) }^{\mathrm{a}}\end{array}$ & $\begin{array}{c}0,22 \\
--\end{array}$ & $\begin{array}{l}0,15 \\
-\ldots\end{array}$ & $\begin{array}{c}0,52 \\
++\end{array}$ & $\begin{array}{l}0,49 \\
+\end{array}$ & $\begin{array}{c}0,56 \\
+\end{array}$ \\
\hline $\begin{array}{l}\text { Combretum micranthum } \\
\text { (sub-adultes) }^{\mathrm{a}}\end{array}$ & $\begin{array}{l}0,02 \\
\text { NS }\end{array}$ & $\begin{array}{l}0,02 \\
\text { NS }\end{array}$ & $\begin{array}{l}0,05 \\
\text { NS }\end{array}$ & $\begin{array}{l}0,09 \\
\text { NS }\end{array}$ & $\begin{array}{l}0,19 \\
+\end{array}$ \\
\hline $\begin{array}{l}\text { Combretum micranthum } \\
\text { (adultes) }^{\mathrm{a}}\end{array}$ & $\begin{array}{l}0,02 \\
-\cdots\end{array}$ & $\begin{array}{l}0,05 \\
-\cdots\end{array}$ & $\begin{array}{l}0,07 \\
-\cdots\end{array}$ & $\begin{array}{l}0,49 \\
+++\end{array}$ & $\begin{array}{l}0,52 \\
+++\end{array}$ \\
\hline $\begin{array}{l}\text { Combretum micranthum } \\
\text { (adultes et sub-adultes } \\
\text { morts) }^{\mathrm{a}}\end{array}$ & $\begin{array}{c}0,01 \\
-\end{array}$ & $\begin{array}{l}0,03 \\
\text { NS }\end{array}$ & $\begin{array}{l}0,05 \\
\text { NS }\end{array}$ & $\begin{array}{l}0,04 \\
\text { NS }\end{array}$ & $\begin{array}{l}0,04 \\
\text { NS }\end{array}$ \\
\hline $\begin{array}{l}\text { Gardenia sokotensis } \\
\text { (adultes et sub-adultes) }^{\mathrm{b}}\end{array}$ & 0,11 & 0,04 & $\begin{array}{c}0,49 \\
+\end{array}$ & $\begin{array}{l}0,68 \\
++\end{array}$ & $\begin{array}{l}0,27 \\
\text { NS }\end{array}$ \\
\hline $\begin{array}{l}\text { Pterocarpus lucens } \\
\text { (jeunes) }^{\mathrm{b}}\end{array}$ & $\begin{array}{l}0,38 \\
\text { NS }\end{array}$ & $\begin{array}{l}0,2 \\
\cdots\end{array}$ & $\begin{array}{l}0,57 \\
++\end{array}$ & $\begin{array}{l}0,44 \\
\text { NS }\end{array}$ & $\begin{array}{l}0,67 \\
++\end{array}$ \\
\hline $\begin{array}{l}\text { Pterocarpus lucens } \\
\text { (sub-adultes) }^{\mathrm{b}}\end{array}$ & $\begin{array}{l}0,36 \\
\text { NS }\end{array}$ & $\begin{array}{l}0,04 \\
\ldots\end{array}$ & $\begin{array}{l}0,68 \\
++\end{array}$ & $\begin{array}{l}0,57 \\
\text { NS }\end{array}$ & $\begin{array}{l}0,55 \\
\text { NS }\end{array}$ \\
\hline $\begin{array}{l}\text { Pterocarpus lucens } \\
\text { (adultes) }\end{array}$ & $\begin{array}{c}0,18 \\
-\end{array}$ & $\begin{array}{c}0,21 \\
-\end{array}$ & $\begin{array}{l}0,58 \\
\text { NS }\end{array}$ & $\begin{array}{l}0,55 \\
\text { NS }\end{array}$ & $\begin{array}{l}0,58 \\
\text { NS }\end{array}$ \\
\hline
\end{tabular}

Pour chaque cellule du tableau, le nombre de placettes occupées est testé par rapport au nombre attendu, sous l'hypothèse d'une indépendance entre la présence de la classe d'individus considérée et de la localisation dans le motif. Les effectifs observés significativement supérieurs aux effectifs attendus sont représentés par $+(p<0,01),++$ $(\rho<0,001)$ et $+++(p<0,000001)$. Les effectifs significativement inférieurs aux effectifs attendus sont représentés avec la symbolique inverse. ${ }^{a}$ Traitement effectué sur les petites placettes (700). ${ }^{\text {b }}$ Traitement effectué sur les grandes placettes (224). 
variables explicatives utilisées dans la régression (de l'ordre de 60, variable selon le type de placettes). L'effet d'interaction est surtout fort pour les jeunes individus, dans le cas des espèces dominantes, mais aussi dans celui d'espèces secondaires qui étaient mal expliquées par les deux modèles précédents. C'est le cas d'Acacia ataxacantha, de Grewia flavescens et, à un moindre degré, de Boscia senegalensis,

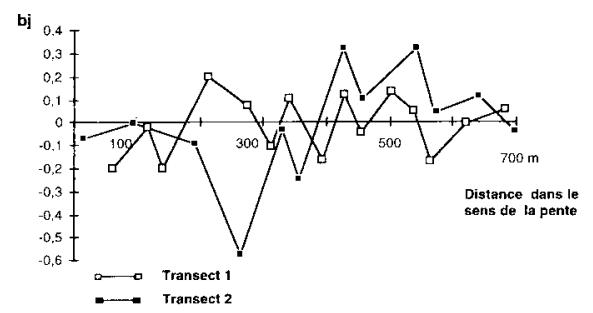

Fig 7. Coefficients bj du modèle «B/A fonction de la position du centre des motifs le long de la toposéquence. Cas des adultes de $C$ micranthum. Ces coefficients expriment la variabilité de l'effectif entre les motifs, la structure interne des motifs ayant déjà été prise en compte (modèle A).
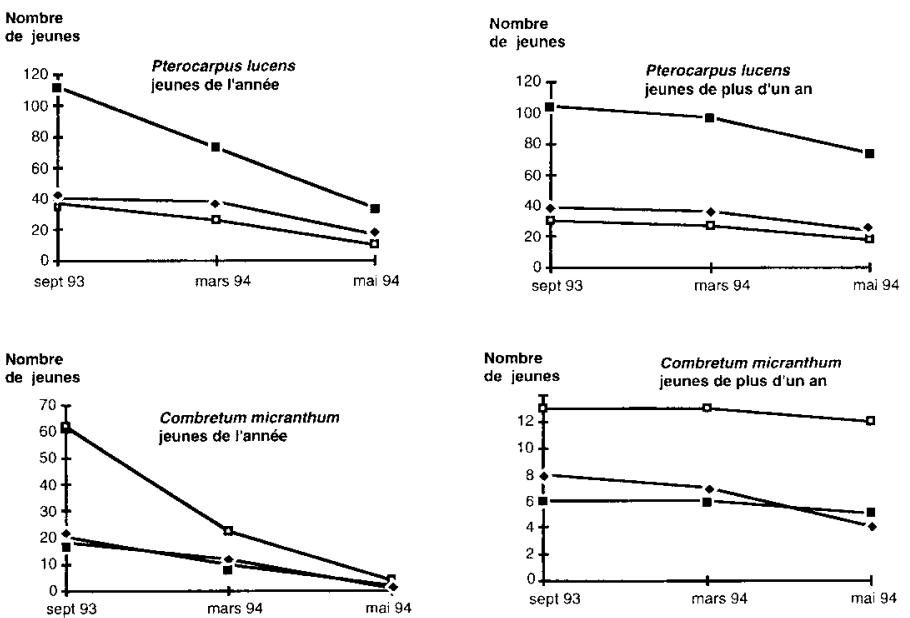

Fig 8. Évolution des effectifs de jeunes individus, pour les deux espèces dominantes, entre septembre 1993 et mai 1994. avec des coefficients de détermination de $0,5,0,29$ et 0,21 , respectivement ; ''interaction est forte aussi pour les sub-adultes de $C$ micranthum. Néanmoins, du fait des faibles densités de ces catégories, il ne semble pas pertinent de pousser plus avant l'analyse. En revanche, pour les jeunes des espèces dominantes, une analyse des coefficients $c k$ du modèle de régression montre que l'effet d'interaction est très largement dû à des variations dans la frange inférieure du fourré : il y a des motifs où celle-ci est bien pourvue en jeunes individus et d'autres où elle est pauvre.

\section{Survie des jeunes individus}

Les résultats de la figure 8 permettent d'apprécier les taux de survie, des jeunes des deux espèces dominantes, durant la saison sèche 1993-94. Le taux de survie des jeunes de l'année est nettement plus fort pour Plucens (en moyenne $32 \%$ ) que pour $C$ micranthum (5\%); en ce qui concerne les jeunes de plus d'un an, le taux de survie de $C$ micranthum est mal connu, du fait du petit nombre d'individus sur les placettes permanentes; il s'établit entre 60 
et $80 \%$, ce qui est du même ordre de grandeur que ceux de $P$ lucens $(67 \%)$. Pour la survie des jeunes individus, la première saison sèche est bien plus critique que les suivantes. Globalement, les taux de survie sont peu variables selon l'élément de la bande de végétation considéré ; sur l'ensemble de la saison, aucune différence n'est significative à $5 \%$ (test exact de probabilité de Fisher; Siegel, 1956, p 106). En revanche, dans le fourré, les jeunes $C \mathrm{mi}$ cranthum se signalent par une forte mortalité durant la première partie de la saison sèche. Les bandes peu couvertes n'ont pas fait l'objet d'une implantation systématique de placettes permanentes, du fait d'un peuplement de jeunes individus trop irrégulier. Néanmoins, en 1992, le rapport entre jeunes Pterocarpus de plus d'un an et de l'année y était de 0,53 (tableau II), soit une valeur assez proche de celle trouvée pour l'ensemble des éléments du motif $(0,47)$; il paraît donc raisonnable de penser que les taux de survie n'y sont pas très différents de ceux rencontrés dans les bandes de végétation.

Tous ces résultats laissent penser que les grands traits des répartitions spatiales des jeunes individus, telles que décrites à partir de l'inventaire de 1992, se maintiennent au-delà des premières saisons sèches: les effectifs sont fortement réduits, mais le " patron " de répartition perdure dans ses grandes lignes. D'après ce qui précède, on peut estimer la mortalité du fait des deux premières saisons sèches à $80 \%$ pour $P$ lucens et à $97 \%$ pour $C$ micranthum.

\section{DISCUSSION}

\section{Une forte emprise végétale et des structures atténuées}

Beaucoup des résultats décrits précédemment dérivent, en fait, de la forte emprise du couvert végétal. L'examen de la figure 2 permet de se rendre compte que les sols nus sont moins étendus que le couvert vé- gétal. De plus, les bandes ou les taches constituant ce dernier sont souvent reliées entre elles par des connexions plus ou moins larges ; la structure d'ensemble, soit l'alternance de bandes couvertes et de bandes nues, apparaît atténuée dès qu'elle est considérée à grande échelle. Cette constatation éclaire les résultats de l'analyse des variogrammes : une périodicité de l'ordre de $60 \mathrm{~m}$ ne s'observe que pour peu de catégories d'individus. La principale exception est constituée par les adultes de $C$ micranthum; c'est cette catégorie qui crée la structure d'ensemble, du point de vue des peuplements ligneux (dont elle représente près des deux tiers de la surface terrière). Pour la plupart des autres espèces (à l'exception de $G$ sokotensis), les individus peuvent être dispersés assez loin des franges des fourrés, et leurs répartitions sont peu marquées par cette structure d'ensemble. En ce qui concerne $P$ lucens (individus adultes), celle-ci ne se manifeste que par des " trous " correspondant à certaines bandes peu couvertes. Pour Boscia senegalensis, la densité des adultes est à peine plus faible dans les bandes peu couvertes.

C'est le couvert des herbacées qui a la plus grande extension spatiale ; il se répartit principalement sur le pourtour des ligneux et, notamment, sur les deux franges des bandes de végétation (même si les surfaces en amont sont plus importantes). Dans son ensemble, ce couvert s'est considérablement étendu depuis la fin des années 1980 et cette extension ouvre la voie à l'installation de jeunes individus ligneux, en grande majorité des $P$ lucens.

\section{Des évolutions limitées}

Le devenir de la structure en bandes actuelle renvoie en priorité à la cinétique du peuplement de $C$ micranthum. Celle-ci apparaît comme très lente. En effet, si les jeunes individus sont nombreux certaines années (comme celles observées), très peu d'entre eux survivent à la première saison sèche ; ces survivants sont principale- 
ment localisés dans le fourré et sur ses abords immédiats. D'autre part, la mortalité actuelle des adultes n'a pas de logique spatiale bien évidente (tableau III). Tout cela paraît correspondre plus à un lent renouvellement des implantations existantes qu'à une modification de celles-ci. En particulier, ne s'observe aucun front d'extension sur les franges amont des fourrés. (Ce qui, pour cette espèce, n'a d'ailleurs jamais été explicitement décrit dans la littérature.) L'implantation de ces peuplements ne semble pas avoir été grandement modifiée par la dernière sécheresse (1967-1985) : la comparaison des photographies aériennes au $1 / 50000^{\circ}$ (prises en 1955 et 1984), certes forcément imprécise, ne montre pas de grandes différences, et les formes des fourrés les plus caractéristiques se sont conservées. Qui plus est, sur le terrain, ne s'observent que très peu de souches d'arbustes morts (" chicots"), qui sont décrits comme très fréquentes, dans les franges inférieures des fourrés, au $\mathrm{Ni}$ ger (Ambouta, 1984).

Pour $P$ lucens, l'autre espèce dominante, les cinétiques sont plus nettes ; en particulier, une proportion notable de jeunes individus survit aux premières saisons sèches. Ils s'observent dans des situations variées, dès lors qu'une certaine rugosité de la surface du sol (herbe, bois mort, ornière...) permet le piégeage des diaspores. À l'intérieur d'une bande de végétation, les taux de survie sont peu variables, mais la distribution initiale des semis de l'année donne un avantage à la frange supérieure, alors que les franges inférieures sont, néanmoins, bien pourvues. Cet avantage initial doit-il se traduire, à moyen terme, par un peuplement plus dense ? Cela ne paraît pas évident, si l'on considère que les sub-adultes, à savoir la régénération " durable " de l'espèce au cours de la dernière décennie, ne sont guère plus nombreux à l'amont qu'à l'aval (quoique mieux répartis). Faut-il considérer que le retour à de meilleures précipitations - à supposer qu'il perdure - puisse changer ce schéma, en per- mettant à des peuplements plus denses de se développer dans les franges supérieures ? C'est là encore discutable, car l'avantage de ces dernières, en termes de bilan hydrique, devrait être plus marqué en période sèche. En l'état actuel de nos connaissances, l'hypothèse d'une extension, relativement isotrope sur tout le pourtour des bandes de végétation, est encore la moins coûteuse en suppositions. De toutes façons, $P$ lucens donne toujours, à l'état adulte, des peuplements faiblement agrégatifs (comparés à d'autres espèces), et ne peut pas, à lui seul, déterminer une extension des fourrés (sensu stricto). Compte tenu de l'état actuel des peuplements, une telle extension ne pourrait être le fait que de $C$ micranthum, donc soit très lente, soit limitée à des circonstances exceptionnelles, encore jamais décrites.

\section{Spécificité des fourrés étudiés}

Ce qui vient d'être dit correspond à un tableau sensiblement différent de ce qui a pu être décrit pour d'autres formations végétales en bandes et, en particulier, pour les exemples du Niger et du Nigéria, qui sont les plus facilement comparables (Ambouta, 1984 ; White, 1970 et 1971). Ces auteurs concluent à une vraisemblable montée des fourrés du fait :

- d'une très forte colonisation de la frange supérieure, due principalement à Guiera senegalensis ;

- d'une régression de la frange inférieure, du fait de la mortalité de $C$ micranthum (cette observation n'est rapportée que par Ambouta et concerne le début des années 1980, soit le point culminant de la période sèche).

Le moteur d'une montée significative du fourré ne peut être assuré que par une espèce, arbustive ou buissonnante, bénéficiant d'aptitudes fortes à la régénération par graines et à la reproduction végétative. Ces conditions sont remplies par Guiera senegalensis, qui est effectivement décrit comme responsable de l'extension en 
amont des fourrés dans les "brousses tigrées " nigériennes. Au nord Yatenga, cette espèce a effectivement une préférence pour les franges supérieures (tableau II) ; elle est cependant relativement rare (20 adultes/ha).

\section{CONCLUSION}

Les peuplements ligneux des fourrés tigrés du nord Yatenga présentent une structure horizontale complexe, pas toujours aussi simple à appréhender que ce que l'on pourrait, a priori, supposer dans ce genre de formation. Ainsi, la structure horizontale ne peut qu'imparfaitement être réduite à une juxtaposition de motifs, tous identiques entre eux. Cela semble découler, d'une part de précipitations relativement fortes, autorisant un couvert végétal étendu, d'autre part de la présence de $P$ lucens, comme espèce co-dominante. Dans le paysage, seules des conditions de pente très particulières déterminent, localement, l'existence d'une structure horizontale, perçue par l'œil comme tigrée. Tout se passe comme si, à la latitude envisagée (env $14^{\circ} 00 \mathrm{~N}$ ), le fourré tigré n'était qu'une forme limite de fourrés " tachetés ", rendue possible par des conditions topographiques spécifiques, et confortée par des périodes de sécheresse.

De ce point de vue, il serait intéressant de reproduire la démarche d'étude plus au nord (aux environs de $15^{\circ} 00 \mathrm{~N}$ ), à une latitude où les fourrés tigrés sont décrits comme fréquents (Boudet, 1972 ; Leprun 1992), mais où des descriptions fines font défaut. Ceci dans l'objectif de situer la position latitudinale d'un éventuel optimum des structures de végétation en bandes, dans l'espace soudano-sahélien.

\section{REMERCIEMENTS}

Ce travail a été appuyé par le CNRS dans le cadre du programme "Savanes à long terme " (Salt), et de l'action incitative "Systèmes intégrés ". Nous sommes particulièrement rede- vables à O Planchon (Orstom) pour son accueil à la station de Bidi, au $\operatorname{Pr} M$ Godron, pour sa critique du manuscrit initial, et à C Millier (Engref) pour l'appui apporté lors de la révision de l'article.

\section{RÉFÉRENCES}

Ambouta K (1984) Contribution à l'édaphologie de la brousse tigrée de l'ouest nigérien. Thèse doct-ing, université de Nancy-I, $116 \mathrm{p}$

Bachacou J, Decourt N (1976) Étude de la compétition dans les plantations régulières à l'aide de variogrammes. Ann sci For 33, 177-198

Bariteau M (1992) Régénération naturelle de la forêt tropicale humide de Guyane : étude de la répartition spatiale de Qualea rosea Aublet, Eperua FalcataAublet et Symphonia globulifera Linnaeus f. Ann $\mathrm{SCl}$ For 49, 359-382

Boaler SB, Hodge CAH (1964) Observations of vegetation arcs in the Northern region, Somali Republic. $J$ Ecol 52, 511-544

Boudet G (1972) Désertification de l'Afrique tropicale sèche. Adansonia série 2, 12, 505-524

Casenave A, Valentin C (1989) Les états de surface de la zone sahélienne. Influence sur l'infiltration, Orstom, Bondy, $230 \mathrm{p}$

Chessel D (1978) Description non paramétrique de la dispersion spatiale des individus d'une espèce. In : Biométrie et écologie (JM Legay, R Tomassone, eds), Société française de biométrie, $n^{\circ} 1,45-136$

Clos-Arceduc M (1956) Étude sur photographies aériennes d'une formation végétale sahélienne : la brousse tigrée. Bulletin de I'IFAN, Ser A, 18,677-684

Cornet A, Delhoume JP, Montana C (1988) Dynamics of striped vegetation patterns and water balance in the Chihuahuan desert. In : Diversity and Pattern in Plant Communities (HJ During, MA Werger, HJ Willems, eds), SPB Academic Publishing, La Haye, 221-231

Couteron P, D'aquino P, Ouedraogo IMO (1992) Pterocarpus lucens Lepr au Nord-Yatenga (Burkina Faso). Intérêt pastoral et état actuel des peuplements. Revue Elev Méd Vet Pays Trop, 45, 179-190

Cressie NAC (1993) Statistics for spatial data. Wiley series in probability and mathematical statistics, John Wiley \& Sons, New York, $900 p$

Grace J (1989) Pattern analysis for forest ecology. In : Unification of European Forest Pattern Research ( $\mathrm{P}$ Schmidt, RAA Oldeman, A Teller, eds), Pudoc, Wageningen, 23-36

Greig-Smith $P(1979)$. Patterns in vegetation. J Ecol 67 , 755-779

Guillet $F$ (1992) Étude et modélisation hydrologique d'un bassin versant de la zone sahélo-soudanienne au Burkina Faso. Thèse, université Paris-V|, $266 p$

Lebreton JD, Roux M, Bacou AM, Banco G (1990) Bioméco (Biométrie-écologie), version 3.9, software of statistical ecology for PC, CNRS, Montpellier, $25 \mathrm{p}$

Leprun JC (1992) Étude de quelques brousses tigrées sahéliennes : structure, dynamique, écologie. In : 
L'aridité, une contrainte au développement (E Le Floc'h, M Grouzis, A Cornet, JC Bille, eds), Orstom, Bondy, 221-244

Matheron $\mathrm{G}$ (1970) La théorie des variables régionalisées et ses applications. Les cahiers du centre de morphologie mathématique de Fontainebleau. Fasc 5 , Ecole nationale supérieure des mines, Paris, $212 \mathrm{p}$

Mauchamp A, Rambal S, Lepart J (1994) Simulating the dynamics of a vegetation mosaic : a spatialized functional model. Ecol Modelling 71, 107-130

Montana C, Lopez-Portillo J, Mauchamp A (1990) The response of two woody species to the conditions created by a shifting ecotone in an arid ecosystem. $J$ Ecol 78, 789-798

Ouedraogo $P$ (1992) Rôle des termitières dans la dynamique d'un faciès de brousse tigrée soudano-sahé- lienne : cas de la région de Bidi au Nord-Yatenga (Burkina Faso). Mémoire de DEA, université Paris$\mathrm{VI}, 49 \mathrm{p}$

Serpantié G, Tezenas du Montcel L, Valentin C (1992) La dynamique des états de surface d'un territoire pastoral soudano-sahélien. Conséquences et propositions. In : L'aridité, une contrainte au développement ( $\mathrm{E}$ Le Floc'h, M Grouzis, A Cornet, JC Bille, eds), Orstom, Bondy, 419-447.

Siegel S (1956) Non-parametric Tests. McGraw-Hill series in psychology, McGraw-Hill, New York, 312p

White LP (1970) Brousse tigree patterns in Southern Niger. $J$ Ecol $58,549-553$

White LP (1971) Vegetation stripes on sheet wash surfaces. J Ecol $59,615-622$ 